\title{
ON FOURIER COEFFICIENTS OF SIEGEL MODULAR FORMS OF DEGREE TWO
}

\author{
BY ERIK A. LIPPA ${ }^{1}$
}

Communicated by Olga Taussky Todd, March 12, 1973

\begin{abstract}
Siegel modular forms $f$ of degree two are considered which satisfy: (1) the Fourier coefficients $b_{f}(R)$, for $R$ a positive definite, semi-integral, primitive matrix, are solely a function of $\operatorname{det}(R)$; and (2) $f$ is an eigenform for the Hecke algebra whose eigenvalues satisfy certain relationships. For such forms, results about multiplicative relationships and asymptotic growth are given, and formulae are given for $b_{f}(R)$ with $R$ arbitrary in terms of $b_{f}(T)$ with $\operatorname{det}(2 T)$ squarefree.
\end{abstract}

Hecke operators play a vital role in investigating multiplicative relations among Fourier coefficients of modular forms of one complex variable. In this paper, we show that, for a certain class of Siegel modular forms of degree two, Hecke operators play a similar role in determining relations among Fourier coefficients.

Let $f(Z)$ be a Siegel modular form of degree two and weight $w$. Then $f(Z)$ has a Fourier expansion of the form $f(Z)=\sum_{R \geqq 0} b_{f}(R) e(R Z)$, where $Z$ is a point in the Siegel upper half plane of degree two, $R$ runs through all positive semidefinite, semi-integral $2 \times 2$ matrices, and $e(R Z)=$ $\exp [2 \pi i \cdot \operatorname{Trace}(R Z)]$. If $R=\left(\begin{array}{cc}a & b \\ b & c\end{array}\right)$, with $a, c, 2 b$ integers, then we set $\operatorname{gcd}(R)=\operatorname{gcd}(a, c, 2 b)$. We will denote the determinant of a matrix $A$ by $|A|$.

We now define the Hecke operators (degree two) on the space $\mathscr{F}_{w}$ of all Siegel modular forms of degree two and weight $w$. Let

$$
J=\left(\begin{array}{cc}
0 & I_{2} \\
-I_{2} & 0
\end{array}\right), \quad \mathscr{L}(n)=\left\{M \in G L(4, Z): M^{t} J M=n J\right\} .
$$

For $f$ in $\mathscr{F}_{w}, n$ a positive integer, and $M$ in $\mathscr{L}(n)$, we write $M$ in blocks of $2 \times 2$ matrices as $M=\left(\begin{array}{cc}A & B \\ C & D\end{array}\right)$ and define

$$
\left(\left.f\right|_{M}\right)(Z)=|M|^{w / 2}|C Z+D|^{-w} f\left[(A Z+B)(C Z+D)^{-1}\right] .
$$

Noting that one can write $\mathscr{L}(n)=\bigcup_{A} \mathscr{L}(1) A$, a finite, disjoint union, we define the unnormalized Hecke operator $T(n): \mathscr{F}_{w} \rightarrow \mathscr{F}_{w}$ as $\left.f\right|_{T(n)}=\left.\sum_{A} f\right|_{A}$.

AMS (MOS) subject classifications (1970). Primary 10D20, 32N15, 42A16.

${ }^{1}$ The main body of this work was carried out at Oxford University under a NATO Postdoctoral Fellowship. 
Formally one has [3, Satz 2],

$$
\sum_{n=1}^{\infty} T(n) n^{-s}=\prod_{p} T\left(p ; p^{-s}\right)
$$

where the product is over all primes. In [2, Theorem 2] we show that formally

$$
\begin{aligned}
T(p ; x)=(1 & \left.-p^{2} x^{2}\right) \\
& \times\left[\left(1-p^{2} x^{2}\right)\left(1-p^{4} x^{2}\right)-T(p)\left(x+p^{3} x^{3}\right)+p W(p) x^{2}\right]^{-1},
\end{aligned}
$$

where $W(p)=(1 / p)\left[T(p)^{2}-T\left(p^{2}\right)\right]+p^{3}$. (See also [8, Theorem 2] and [7, Appendix I, Equation 21].)

We shall be considering $f$ in $\mathscr{F}_{w}$ which are eigenforms for all the $T(n)$. We shall denote its eigenvalues under $T(p)$ and $W(p)$ by $\tau_{p, f}$ and $\lambda_{p, f}$, respectively. In particular, we shall be interested in forms $f$ satisfying two additional properties, viz.:

Property I. For $R$ primitive (i.e. $\operatorname{gcd}(R)=1) b_{f}(R)$ is solely a function of $|R|$ and

Property II $(p)$. For the prime $p, \lambda_{p, f}=(p+1) \tau_{p, f}$.

Property $I$ is true for Eisenstein series [4, Satz 1] and appears to be probable for the unique cusp form of weight 10 on the basis of numerical data $[6, p .30]$ and for the unique cusp form of weight 12 for theoretical considerations. Property II $(p)$ is true for all primes $p$ for Eisenstein series and we shall show (Theorem 4) that, in a number of cases, Property I implies Property II $(p)$. (This is true in particular for all $p$ for the cusp form of weight 12.)

The appearance of Property $\mathrm{II}(p)$ is related to the factorization of $T(p)$ and $W(p)$ as [2, p. 28 and Lemma 9]:

$$
(p+1) T(p)=L(p) \cdot M(p) \text { and } W(p)=L(p) \cdot V(p) \cdot M(p),
$$

where $L(p), V(p)$, and $M(p)$ are Hecke operators of the Koecher type $[1$, p. 361]. Here, $V(p)$ is an involution.

We prove:

THEOREM 1. Let $f$ in $\mathscr{F}_{w}$ be an eigenform for the Hecke algebra and suppose $f$ has Property I. Then $b_{f}(R)$ depends solely on $\operatorname{gcd}(R)$ and $|R|$.

Note. Theorem 1 for Eisenstein series follows from a correction of $[4$, Satz 2] in [5].

Let

$$
b_{f}\left[a\left(r_{1}, r_{2}, r_{3}\right)\right]=b_{f}\left[a\left(\begin{array}{cc}
r_{1} & r_{3} / 2 \\
r_{3} / 2 & r_{2}
\end{array}\right)\right],
$$

for $a, r_{1}, r_{2}, r_{3}$ integers; $p$ and $q$ denote distinct primes; $y, z, s$, and $t$ denote formal power series variables; $x_{p, w}=p^{w-2} ;(A / B) \equiv$ Jacobi symbol, 
$((2 A+1) / 2) \equiv 1$, for integers $A$ and $B$

$$
\begin{aligned}
\Delta_{f}(p ; t) & =1+\left(p+1-p^{-1} \tau_{p, f}\right) x_{p, w} t+p x_{p, w}^{2} t^{2} ; \\
D_{f}(p ; y, z) & =\left(1-p x_{p, w} y\right) \Delta_{f}(p ; y) \Delta_{f}\left(p ; z^{2}\right) ; \\
\beta_{w}(p ; y, z) & =\left(1-p x_{p, w}^{2} y z^{2}\right) ; \\
\gamma_{f}(M, p ; y, z) & =\left[1-(-M / p) x_{p, w} y\right] \beta_{w}(p ; y, z)-(-M / p) x_{p, w} z^{2} \Delta_{f}(p ; y) ;
\end{aligned}
$$

and for positive integers $C$ and $T$ with $(C T, p q)=1$, we set $H_{f}(p, q ; C, T ; y, z, s, t)$

$$
=\sum_{m, n, a, b=0}^{\infty} b_{f}\left[C p^{n} q^{a}\left(1, p^{m} q^{b} T, 0\right)\right] y^{n} z^{m} s^{a} t^{b} D_{f}(p ; y, z) D_{f}(q ; s, t) .
$$

We prove:

THEOREM 2. Let $p$ and $q$ be distinct odd primes, $C$ and $T$ positive integers such that $(C T, p q)=1$. Let $f$ in $\mathscr{F}_{w}$ be an eigenform for the Hecke algebra and suppose $f$ has Properties $\mathrm{I}, \mathrm{II}(p)$, and $\mathrm{II}(q)$. Then

$$
\begin{aligned}
H_{f}(p, q ; C, T ; y, z, s, t)= & \gamma_{f}(T, p ; y, z) \gamma_{f}(T, q ; s, t) b_{f}[C(1, T, 0)] \\
& +\gamma_{f}(p T, q ; s, t) \beta_{w}(p ; y, z) z b_{f}[C(1, p T, 0)] \\
& +\gamma_{f}(q T, p ; y, z) \beta_{w}(q ; s, t) t b_{f}[C(1, q T, 0)] \\
& +\beta_{w}(p ; y, z) \beta_{w}(q ; s, t) z t b_{f}[C(1, p q T, 0)] .
\end{aligned}
$$

THEOREM 3. Let $p$ be an odd prime. Let $f$ in $\mathscr{F}_{w}$ be an eigenform for the Hecke algebra and suppose $f$ has Properties $\mathrm{I}, \mathrm{II}(2)$, and $\mathrm{II}(p)$. Let $C$ and $T$ be positive integers such that $(C T, 2 p)=1$. Then

$$
\begin{aligned}
H_{f}(p, 2 ; C, T ; y, z, s, t)= & \beta_{w}(2 ; s, t) \gamma_{f}(T, p ; y, z) b_{f}[C(1, T, 0)] \\
+ & \beta_{w}(2 ; s, t) \gamma_{f}(2 T, p ; y, z) t b_{f}[C(1,2 T, 0)] \\
+ & \beta_{w}(2 ; s, t) \beta_{w}(p ; y, z) z b_{f}[C(1, p T, 0)] \\
+ & \beta_{w}(2 ; s, t) \beta_{w}(p ; y, z) z t b_{f}[C(1,2 p T, 0)] \\
+ & {[1-(-1 / p T)] x_{2, w} } \\
& \cdot\left\{\gamma_{f}(1,2 ; s, t)-\beta_{w}(2 ; s, t)\right\} \\
& \cdot \beta_{w}(p ; y, z) z b_{f}\left[C\left(\frac{1}{4}(p T+1), 1,1\right)\right] \\
+ & {[1-(-1 / T)] x_{2, w}\left\{\gamma_{f}(1,2 ; s, t)-\beta_{w}(2 ; s, t)\right\} } \\
& \gamma_{f}(T, p ; y, z) b_{f}\left[C\left(\frac{1}{4}(T+1), 1,1\right)\right] .
\end{aligned}
$$

If $M$ and $N$ are positive integers such that $M \equiv 3(\bmod 4)$ and $N \equiv 1$ $(\bmod 2)$, then:

$b_{f}[N(1, M, 0)]$

$$
=\left\{2^{-1} x_{2, w} \tau_{2, f}-[3+(2 / M)] x_{2, w}\right\} b_{f}\left[N\left(\frac{1}{4}(M+1), 1,1\right)\right] .
$$


THEOREM 4. Let $f$ in $\mathscr{F}_{w}$ be an eigenform for the (degree two) Hecke algebra. Let

$$
f\left[\left(\begin{array}{cc}
z_{1} & 0 \\
0 & z_{2}
\end{array}\right)\right]=\sum_{i} g_{i}\left(z_{1}\right) g_{i}\left(z_{2}\right),
$$

where the $g_{i}$ are modular forms of one complex variable of weight $w$ which are eigenforms for the (degree one) Hecke algebra.

(1) If $f$ has Property I and $b_{f}(1,1,0) \neq 0$, then $f$ has Property II(2).

(2) If $p$ is an odd prime, $f$ has Property I, and $[p-(-3 / p)] b_{f}(1,1,0)+$ $[p-(-1 / p)] 2 b_{f}(1,1,1) \neq 0$, then $f$ has Property $\mathrm{II}(p)$.

Let

$$
\begin{aligned}
b_{f}(\langle R\rangle) & =b_{f}(1,|R|, 0), & & |R| \in \boldsymbol{Z}, \\
& =b_{f}\left(|R|+\frac{1}{4}, 1,1\right), & & |R| \notin Z .
\end{aligned}
$$

We shall prove the following theorem which includes some of the conjectures of [6]. The proof of Theorem 5, Parts 2a, b below for Eisenstein series will appear shortly in [5] via very different methods.

THEOREM 5. (1) If $f$ is the normalized Eisenstein series of weight $w$ then $f$ has Property I and Property II $(p)$ for all primes $p$. In particular

$$
\tau_{p, f}=p x_{p, w}^{-1}\left(1+p x_{p, w}\right)\left(1+x_{p, w}\right) .
$$

Also, for any $\varepsilon>0$ and $R$ positive definite,

$$
b_{f}(R)=0\left(|R|^{w-(3 / 2)+\varepsilon}\right)
$$

where the implied constant depends only on $w$ and $\varepsilon$.

(2) Let $f$ in $\mathscr{F}_{w}$ be an eigenform for the Hecke algebra and suppose $f$ has Property I and Property II $(p)$ for all primes $p$ and $\tau_{2, f} \neq 4,8$. Then, for semi-integral, positive definite $R$,

and

$$
b_{f}(R)=\sum_{0<d \mid \operatorname{gcd}(R)} d^{w-1} b_{f}(\langle R / d\rangle)
$$

for $S$ a primitive matrix and relatively prime positive integers $M$ and $N$.

If, in addition, $\left|p+1-p^{-1} \tau_{p, f}\right| \leqq 2 \sqrt{ } p$ for all primes $p$, then for any $\varepsilon>0$ and $R$ positive definite

$$
b_{f}(R)=0\left([\operatorname{gcd}(R)]^{1 / 2}|R|^{(2 w-3) / 4+\varepsilon}\right),
$$

where the implied constant depends only on $f$ and $\varepsilon$.

Note. If $\chi_{12}$ and $\chi_{10}$ are the unique normalized cusp forms of weight 12 and 10, respectively, then [6, pp. 37-38] and Theorem 4 imply that 
if $\chi_{10}$ and $\chi_{12}$ have Property I, then:

(a) $\chi_{12}$ has Property II $(p)$ for all primes $p$, and

(b) $\chi_{10}$ has Property $\mathrm{II}(p)$ whenever $(3 / p) \neq 1$ or $p=2$.

\section{REFERENCES}

1. M. Koecher, Zur Operatorentheorie der Modulformen n-ten Grades, Math. Ann. 130 (1956), 351-385. MR 17, 1058.

2. E. Lippa, Hecke operators for modular forms of genus $r$, Ph.D. Dissertation, University of Michigan, Ann Arbor, Mich., 1971.

3. H. Maass, Die Primzahlen in der Theorie der Siegelschen Modulfunktionen, Math. Ann. 124 (1951), 87-122. MR 13, 823.

4. —- Die Fourierkoeffizienten der Eisensteinreihen zweiten Grades, Mat.-Fys. Medd. Dancke Vid. Selsk. 34 (1964), no. 7, 25 pp. MR 30 \# 1985.

5. ——Über die Fourierkoefficienten der Eisenstreinreihen zweiten Grades, Mat.-Fys. Medd. Dancke Vid. Selsk. 38 (1972), no. 14. 13pp.

6. H. L. Resnikoff and R. L. Saldaña, Some properties of Fourier coefficients of Eisenstein series of degree two (to appear).

7. I. Satake, Theory of spherical functions on reductive algebraic groups over P-adic fields, Inst. Hautes Études Sci. Publ. Math. No. 18 (1963), 5-69. MR 33 \# 4059.

8. G. Shimura, On modular correspondences for $\mathrm{SP}(\mathrm{N}, \mathrm{Z})$ and their congruence relations, Proc. Nat. Acad. Sci. U.S.A. 49 (1963), 824-828. MR 28 \# 250.

Department of Mathematics, Purdue University, Lafayette, Indiana 47907 (Current address) 